\section{Estado nutricional e fatores associados ao déficit estatural em crianças menores de cinco anos de comunidades remanescentes de quilombos do Nordeste brasileiro}

\author{
Nutritional status and factors associated with \\ stunting in children under five years of age in \\ maroon communities in Northeast Brazil
}

\section{Estado nutricional y factores asociados al déficit de estatura en niños menores de cinco años originarios de comunidades reminiscentes de quilombos en el Nordeste Brasileño}

Félix de Jesus Neves 1 Aline Alves Ferreira 2 James R. Welch 3

\title{
Resumo
}

Os quilombolas constituem parte das minorias étnico-raciais do país que apresentam iniquidades em saúde, reflexo de um processo histórico de grandes desvantagens socioeconômicas. O objetivo foi avaliar o estado nutricional e fatores associados ao déficit estatural em crianças quilombolas menores de cinco anos residentes em comunidades quilombolas tituladas na Região Nordeste do Brasil. Utilizaram-se dados secundários provenientes da Pesquisa de Avaliação da Situação de Segurança Alimentar e Nutricional em Comunidades Quilombolas Tituladas (2011). Os desfechos de interesse foram $o$ déficit estatural (estatura-para-idade $<-2 z$ ), excesso de peso (peso-para-estatura $>2 z$ ) e o déficit ponderal (peso-para-idade $<-2 z$ ). Foi empregado o teste qui-quadrado para avaliar a significância das diferenças entre as prevalências. A análise multivariada considerou um modelo conceitual hierárquico sobre o déficit estatural. As prevalências de excesso de peso e déficit ponderal foram 2,8\% e 6,1\%, respectivamente. O déficit estatural foi diagnosticado em $14,1 \%$ da amostra. O modelo hierárquico de déficit estatural evidenciou maiores prevalências do agravo entre crianças que não tinham acesso à atenção básica $(R P=1,63$; IC95\%: 1,11; 2,41), à água tratada $(R P=2,09 ;$ IC95\%: $1,42 ; 3,08)$ e que nasceram com baixo peso ( $R P=2,19$; IC95\%: 1,33; 3,61). A elevada prevalência de déficit estatural mostra que a população quilombola no Nordeste apresenta condições de saúde desfavoráveis, sendo reflexo da falta de acesso à atenção básica e das precárias condições de saneamento.

Etnia e Saúde; Grupo com Ancestrais do Continente Africano; Avaliação Nutricional; Desnutrição

\author{
Correspondência \\ J. R. Welch \\ Escola Nacional de Saúde Pública Sergio Arouca, Fundação \\ Oswaldo Cruz. \\ Rua Leopoldo Bulhões 1480, Rio de Janeiro, RJ 21041-210, \\ Brasil. \\ welch@ensp.fiocruz.br \\ 1 Escola Paulista de Medicina, Universidade Federal de São \\ Paulo, São Paulo, Brasil. \\ 2 Instituto de Nutrição Josué de Castro, Universidade Federal do \\ Rio de Janeiro, Rio de Janeiro, Brasil. \\ 3 Escola Nacional de Saúde Pública Sergio Arouca, Fundação \\ Oswaldo Cruz, Rio de Janeiro, Brasil.
}




\section{Introdução}

Uma acelerada transição alimentar e nutricional ocorreu nas últimas décadas no Brasil 1, resultando em um aumento das frequências de sobrepeso e obesidade e na redução da prevalência de desnutrição, especialmente na população infantil. No entanto, as mudanças no perfil de saúde que acompanharam a transição nutricional não atingiram a população brasileira uniformemente. Minorias étnico-raciais continuam a apresentar desvantagens socioeconômicas que se refletem em perfis de morbidade menos satisfatórios, principalmente em relação a agravos nutricionais. Dentre essas minorias, encontram-se os quilombolas 2,3, que vivem em situações particularmente pronunciadas de exclusão social 4 .

As origens da população quilombola estão relacionadas ao período escravocrata, quando as primeiras comunidades foram formadas como estratégia de resistência e busca de autonomia 5 . Reconhecidos na Constituição Federal como "remanescentes das comunidades dos quilombos", formam um segmento da população brasileira que possui sua própria identidade territorial e étnica ${ }^{6}$, relacionada a aspectos históricos e sociopolíticos que pautaram a luta por reconhecimento 7 .

São povos que enfrentam cotidianamente a discriminação étnico-racial. Este é um fator importante na determinação das condições de saúde em geral nessa população. Os processos de exclusão e discriminação impactam negativamente nas condições socioeconômicas, levando a menor acesso à educação e, consequentemente, ao mercado de trabalho e à habitação, o que se reflete em piores perfis de saúde em comparação aos da população geral 8,9.

Até 2016, 1.525 terras quilombolas encontravam-se em processo de titulação e apenas 165 eram tituladas (as quais dão origem a 169 comunidades), sendo a maioria localizada em áreas rurais ou periféricas da Região Nordeste $(58,4 \%)$ 10. A predominância dessas comunidades na zona rural se deve aos fatos de que, no período escravocrata, quando muitos quilombos foram formados, havia a necessidade de isolamento para fins de proteção e de que a subsistência se baseava na pesca e agricultura. Após a abolição, os quilombos passaram a receber ex-escravos, os quais encontravam nessas comunidades a única alternativa para viver mais dignamente 9 .

Diversos estudos têm indicado que a exclusão social é um fator importante para a compreensão do perfil de saúde dos remanescentes de quilombos 9,11,12. Têm sido reportadas condições precárias de habitação, saneamento, acesso limitado aos serviços de saúde, baixa escolaridade, obesidade abdominal em adultos, entre outras situações 12,13,14,15,16,17. Em crianças, também são comumente registradas elevadas prevalências de anemia, enteroparasitoses, diarreia e desnutrição 2,11,18, em um cenário que contribui para grave insegurança alimentar 19.

Dentre os agravos nutricionais na infância, a desnutrição crônica, identificada pelo déficit estatural, tem sido a condição mais observada em minorias étnico-raciais 11,20,21. No contexto brasileiro, o Nordeste tem apresentado historicamente frequências mais elevadas de agravos nutricionais na infância, principalmente de baixa estatura-para-idade 22, o que caracteriza o efeito cumulativo de precárias condições de nutrição e saúde ao longo do tempo. Porém, não se tem um panorama da situação nutricional de crianças quilombolas para a macrorregião como um todo, tampouco foram investigados os fatores associados.

Considerando esse contexto, o presente estudo buscou avaliar o estado nutricional e fatores associados em crianças quilombolas menores de cinco anos residentes em comunidades quilombolas tituladas na Região Nordeste.

\section{Métodos}

Foram utilizados dados secundários derivados da Pesquisa de Avaliação da Situação de Segurança Alimentar e Nutricional em Comunidades Quilombolas Tituladas (Pesquisa de Avaliação), conduzida em 201123 . Essa pesquisa planejou investigar todos os residentes das 177 comunidades quilombolas tituladas no país (segundo cadastro existente em 2009), localizadas em 14 unidades da federação. Foram coletados dados de 40.548 indivíduos, habitantes de 169 comunidades. A redução de 177 para 169 no total de comunidades pesquisadas se deve ao fato de algumas comunidades terem se fundido a outras em anos anteriores à pesquisa 24 . A população analisada no presente estudo incluiu crianças quilombolas menores de cinco anos, residentes em 49 comunidades situadas em cinco Unidades 
Federativas (UF) (Bahia - 6; Maranhão - 22; Pernambuco - 2; Piauí - 17; Sergipe - 2). Os demais estados nordestinos não foram incluídos no estudo original por não abrigarem, à época, comunidades quilombolas com territórios titulados, embora abrigassem diversas comunidades certificadas como remanescentes de quilombos.

Para a coleta de dados, foram utilizados questionários semiestruturados. O primeiro deles, intitulado Questionário Liderança, visou à coleta de informações referentes às características gerais das comunidades, bem como ao seu acesso a políticas e programas sociais, à presença de escolas e serviços de saúde. Esse questionário foi respondido por 162 lideranças comunitárias. O segundo questionário, denominado Características dos Moradores, foi aplicado durante entrevistas domiciliares e incluiu perguntas sobre as características do domicílio, situação socioeconômica, segurança alimentar dos moradores, considerando-se a Escala Brasileira de Insegurança Alimentar e Nutricional (EBIA), assim como dados sobre as condições de saúde das crianças 24 .

A aferição do peso das crianças $<5$ anos foi realizada com balança digital TANITA, modelo HS 302 (https://www.tanita.com), com capacidade para 150kg. Para mensurar o comprimento de crianças < 2 anos, foi utilizado um infantômetro de madeira, desenvolvido especialmente para a pesquisa, com medidas entre $10 \mathrm{~cm}$ e $120 \mathrm{~cm}$. Para as demais crianças, foi utilizado o antropômetro da marca WCS Tecnologia (https://wcsconectologia.com.br/), com precisão de $0,1 \mathrm{~cm}$ e capacidade de $210 \mathrm{~cm}$. A balança e o antropômetro também foram utilizados para aferição do peso e estatura das mães. Maiores detalhes encontram-se disponíveis em outra publicação 23.

Os índices antropométricos foram calculados com o programa WHO Anthro 2011 (https://www. who.int/tools/child-growth-standards/software) 25. Conforme preconizado pela Organização Mundial da Saúde (OMS) 26, adotaram-se os pontos de corte $<-2$ ou $>2$ escores z para definir as condições de déficit ou excesso para os respectivos índices antropométricos. Dessa forma, foram estabelecidas as seguintes condições de interesse: déficit estatural (estatura-para-idade $-\mathrm{E} / \mathrm{I}<-2$ ), déficit ponderal (peso-para-idade $-\mathrm{P} / \mathrm{I}<-2$ ) e excesso de peso (peso-para-estatura $-\mathrm{P} / \mathrm{E}>2$ ). As crianças que apresentaram valores de escores $\mathrm{z}$ biologicamente implausíveis foram excluídas das análises 25 . $\mathrm{O}$ excesso de peso materno foi classificado segundo o índice de massa corpórea (IMC), calculado através da fórmula peso/altura 2 , utilizando também o ponto de corte da OMS: IMC $>25,00 \mathrm{~kg} / \mathrm{m}^{2}$.

Para fins da análise estatística, foram selecionadas variáveis relacionadas a benefícios sociais (Programas Cestas de Alimentos, Cisternas e Bolsa Família), condição socioeconômica (renda, condição ocupacional, saneamento, características do domicílio, escolaridade), aspectos demográficos (UF de residência, sexo, idade, raça/cor), estado nutricional da mãe e condições de saúde da criança (baixo peso ao nascer e relato de diarreia nos sete dias anteriores à entrevista). Também foram analisadas variáveis referentes ao acesso a serviços de saúde, como frequência de visita do agente comunitário de saúde (ACS), do assistente social e acesso à atenção básica (domicílio em área adstrita por equipe de saúde da família - EqSF).

As variáveis contínuas foram classificadas em duas ou mais categorias, assim como foram reagrupadas algumas variáveis categóricas. A "raça/cor" das crianças foi reportada pelos entrevistados (pais ou responsáveis), segundo as categorias branca, preta, amarela, parda e indígena. Para fins desta pesquisa (amarela e indígena), não foram incluídas devido ao reduzido número de observações (21 e 61, respectivamente).

Foi empregado o teste qui-quadrado $\left(\chi^{2}\right)$ para avaliar a significância das diferenças entre as prevalências dos extremos antropométricos, segundo sexo $(\mathrm{p}<0,05)$. As prevalências de déficit estatural foram analisadas segundo indicadores de acesso à saúde e benefícios sociais, socioeconômicos, de escolaridade, de estado nutricional da mãe, demográficos e de saúde da criança.

A associação de cada variável independente com o déficit estatural (desfecho dicotômico) foi analisada utilizando-se regressão de Poisson com variância robusta (análise bivariada). As variáveis com valor de $\mathrm{p}<0,20$ foram selecionadas para a etapa seguinte (análise multivariada). Foram calculadas as razões de prevalência e seus respectivos intervalos de 95\% de confiança (IC95\%), assim como os parâmetros do modelo através do teste estatístico de Wald.

Foi criado um modelo conceitual hierárquico de determinação da desnutrição, para o que se tomaram por base os trabalhos de Olinto et al. 27 e Martins et al. 28. Segundo essa perspectiva, os fatores de acesso à saúde e benefícios sociais apresentariam relação mais distal com o déficit estatural, em comparação às demais variáveis selecionadas para o estudo. Isso porque o acesso a serviços de saúde 
e a programas sociais podem influenciar direta ou indiretamente no tratamento de enfermidades que estão relacionadas ao agravo. Em seguida, o crescimento seria influenciado por fatores socioeconômicos relacionados à renda e às características do domicílio. Os aspectos ligados à escolaridade e ao estado nutricional da mãe foram avaliados no terceiro nível do modelo hierárquico. Finalmente, no quarto nível, foram agrupadas as variáveis demográficas e de estado de saúde da criança, que apresentam forte associação com o surgimento de agravos que constituem fatores de risco para o déficit estatural, como a diarreia e o baixo peso ao nascer 29,30 .

$\mathrm{Na}$ análise multivariada, foi também empregada a regressão de Poisson com variância robusta, seguindo o método de modelagem hierarquizada com inserção manual de variáveis, distribuídas em quatro níveis de acordo com a sua proximidade com o desfecho, considerando um nível de 5\% de significância. Em cada nível, permaneceram as variáveis que apresentaram valor de $\mathrm{p}<0,05$ para o ajuste no nível seguinte. Este procedimento foi aplicado em todos os níveis do modelo hierárquico, implicando no controle das variáveis dos níveis anteriores até o modelo final. Os modelos foram testados e comparados por meio do critério de informação de Akaike (AIC), considerado a qualidade do ajuste do modelo. Foram testadas todas as interações plausíveis e possíveis confundidores.

A sistematização e a análise dos dados foram realizadas utilizando-se os programas IBM SPSS Statistics versão 21.0 (https://www.ibm.com/) e RStudio versão 0.98.1103 (https://www.rstudio. com/products/rstudio/download/).

Por utilizar dados secundários, disponíveis em sítio eletrônico de domínio público (https://aplica coes.mds.gov.br/sagi/pesquisas/lista-s.php?tp=pesquisa\&stk=ano\&id=135), não houve necessidade de submissão do presente estudo ao Comitê de Ética em Pesquisa.

\section{Resultados}

Nos dados relativos à Região Nordeste, constavam originalmente 1.265 crianças $<5$ anos. Desse total, 10,8\% não apresentavam dados de peso, 14,9\%, de estatura, e 10,4\%, de idade. Dessa forma, foi possível analisar os dados relativos a $972(76,8 \%)$ crianças quanto à E/I, 1.029 (81,3\%) segundo o P/I e 1.053 $(83,2 \%)$ em relação ao $\mathrm{P} / \mathrm{E}$.

A prevalência de déficit estatural foi de 14,1\%, e o déficit ponderal atingiu 6,1\% das crianças. O excesso de peso foi observado em 2,8\% das crianças analisadas (Tabela 1). As prevalências entre

\section{Tabela 1}

Prevalências totais e por sexo de crianças quilombolas menores de cinco anos segundo categorias de escore z de indicadores antropométricos, em comunidades quilombolas tituladas na Região Nordeste, Brasil, 2011.

\begin{tabular}{|c|c|c|c|c|c|c|c|}
\hline \multirow[t]{2}{*}{ Indicador } & \multirow[t]{2}{*}{$\mathbf{N}$} & \multicolumn{2}{|c|}{ Déficit } & \multicolumn{2}{|c|}{ Eutrofia } & \multicolumn{2}{|c|}{ Excesso } \\
\hline & & $\mathbf{n}$ & $\%$ & $\mathbf{n}$ & $\%$ & $\mathbf{n}$ & $\%$ \\
\hline Estatura-para-idade & & $p=0,698$ & & $p=0,150$ & & $p=0,683$ & \\
\hline Feminino & 508 & 69 & 13,6 & 426 & 83,9 & 13 & 2,6 \\
\hline Masculino & 464 & 68 & 14,7 & 385 & 83,0 & 11 & 2,4 \\
\hline Total & 972 & 137 & 14,1 & 811 & 83,4 & 24 & 2,5 \\
\hline Peso-para-idade & & $p=0,705$ & & $p=0,160$ & & $p=0,095$ & \\
\hline Feminino & 542 & 33 & 5,9 & 490 & 90,4 & 19 & 3,5 \\
\hline Masculino & 487 & 30 & 6,6 & 447 & 91,8 & 10 & 2,1 \\
\hline Total & 1.029 & 63 & 6,1 & 937 & 91,1 & 29 & 2,8 \\
\hline Peso-para-estatura & & $p=0,602$ & & $p=0,357$ & & $p=0,869$ & \\
\hline Feminino & 543 & 18 & 3,3 & 510 & 93,9 & 15 & 2,8 \\
\hline Masculino & 510 & 15 & 2,9 & 481 & 94,3 & 14 & 2,7 \\
\hline Total & 1.053 & 33 & 3,1 & 991 & 94,1 & 29 & 2,8 \\
\hline
\end{tabular}

Déficit: escore $z<-2$; Eutrofia: escore $z \geq-2$ e $\leq 2$; Excesso: escore $z>2$; $p$ : valor de $p$ referente ao teste $\chi^{2}$ de Pearson. 
os sexos não apresentaram diferenças estatisticamente significantes para déficit estatural (valor de $\mathrm{p}=0,698$ ), déficit ponderal (valor de $\mathrm{p}=0,705$ ) ou excesso de peso (valor de $\mathrm{p}=0,869$ ).

No tocante às variáveis de acesso à saúde e benefícios sociais com significância estatística nas análises brutas (Tabela 2), a prevalência de déficit estatural em domicílios não beneficiados pelo Programa Cisternas foi 2,29 (IC95\%: 1,01; 5,19) vezes aquela observada em domicílios com presença desse benefício. Foram observadas prevalências 77\% maiores (IC95\%: 1,24; 2,53) em domicílios que não recebiam visita frequente do ACS e $64 \%$ maiores (IC95\%: 1,16; 2,31) naqueles nos quais não havia acesso à atenção básica, quando comparados aos domicílios visitados pelo ACS e aos domicílios com acesso à atenção básica, respectivamente.

Em relação às características socioeconômicas e domiciliares (Tabela 3), as prevalências de déficit estatural foram 92\% maiores (IC95\%: 1,30; 2,83) em domicílios que não tinham banheiro, e 99\% maiores (IC95\%: 1,$41 ; 2,80)$ onde não havia água tratada, se comparados aos que tinham banheiro $\mathrm{e}$ água tratada, respectivamente. Ao se compararem domicílios com rede de esgoto adequada e inadequada, observou-se uma prevalência 78\% maior (IC95\%: 1,06; 3,00) nas residências com esgoto a céu aberto ou com outro escoadouro sanitário inadequado. Nos domicílios em que residiam três ou mais crianças < 5 anos, a prevalência foi $54 \%$ maior (IC95\%: 1,01; 2,34) em comparação àqueles com até duas crianças. Além disso, o agravo foi 32\% menos prevalente (IC95\%: 0,46; 0,99) quando o chefe do domicílio estava empregado, em comparação aos domicílios nos quais o chefe estava desempregado.

Tabela 2

Prevalência e razões de prevalência (RP) brutas de déficit estatural em crianças quilombolas $<5$ anos residentes em terras tituladas na Região Nordeste, Brasil, segundo variáveis de acesso à saúde e benefícios sociais (nível 1), 2011.

\begin{tabular}{|c|c|c|c|c|c|c|c|}
\hline \multirow[t]{2}{*}{ Variáveis } & \multicolumn{2}{|c|}{ E/I adequada } & \multicolumn{2}{|c|}{ Déficit estatural } & \multirow[t]{2}{*}{ Valor de $p$ * } & \multirow[t]{2}{*}{ RP (IC95\%) } & \multirow[t]{2}{*}{ Valor de $p$ ** } \\
\hline & $\mathbf{n}$ & $\%$ & $\mathbf{n}$ & $\%$ & & & \\
\hline \multicolumn{8}{|c|}{ Domicílio beneficiado pelo } \\
\hline \multicolumn{8}{|c|}{ Programa Cisternas } \\
\hline Sim & 86 & 93,5 & 6 & 6,5 & 0,041 & Referência (1) & \\
\hline Não & 747 & 85,1 & 131 & 14,9 & & $2,29(1,01 ; 5,19)$ & 0,047 \\
\hline \multicolumn{8}{|c|}{ Domicílio beneficiado pelo } \\
\hline \multicolumn{8}{|c|}{ Programa Bolsa família } \\
\hline $\operatorname{Sim}$ & 709 & 86,0 & 115 & 14,0 & 0,771 & Referência (1) & \\
\hline Não & 122 & 84,7 & 22 & 15,3 & & $1,09(0,69 ; 1,73)$ & 0,697 \\
\hline \multicolumn{8}{|c|}{ Domicílio beneficiado pelo } \\
\hline \multicolumn{8}{|c|}{ Programa Cestas de Alimentos } \\
\hline $\operatorname{Sim}$ & 426 & 88,2 & 57 & 11,8 & 0,047 & Referência (1) & \\
\hline Não & 401 & 83,5 & 79 & 16,5 & & $1,39(0,99 ; 1,96)$ & 0,059 \\
\hline \multirow{2}{*}{\multicolumn{8}{|c|}{$\begin{array}{l}\text { Família recebe visita frequente } \\
\text { do ACS }\end{array}$}} \\
\hline & & & & & & & \\
\hline $\operatorname{Sim}$ & 666 & 87,9 & 92 & 12,1 & $<0,001$ & Referência (1) & \\
\hline Não & 164 & 78,5 & 45 & 21,5 & & $1,77(1,24 ; 2,53)$ & 0,002 \\
\hline \multicolumn{8}{|c|}{ Domicílio com acesso à atenção } \\
\hline \multicolumn{8}{|l|}{ básica } \\
\hline $\operatorname{Sim}$ & 471 & 89,2 & 57 & 10,8 & 0,002 & Referência (1) & \\
\hline Não & 358 & 82,3 & 77 & 17,7 & & $1,64(1,16 ; 2,31)$ & 0,005 \\
\hline \multicolumn{8}{|c|}{$\begin{array}{l}\text { Família atendida por assistente } \\
\text { social ou equipe do CRAS }\end{array}$} \\
\hline $\operatorname{Sim}$ & 194 & 88,6 & 25 & 11,4 & 0,210 & Referência (1) & \\
\hline Não & 625 & 84,9 & 111 & 15,1 & & $1,32(0,86 ; 2,04)$ & 0,204 \\
\hline
\end{tabular}

ACS: agentes comunitários de saúde; CRAS: Centro de Referencia de Assistência Social; E/l: estatura- para-idade; IC95\%: intervalo de 95\% de confiança.

* Teste $\chi^{2}$ de Pearson;

** Teste de Wald. 
Tabela 3

Prevalência e razões de prevalência (RP) brutas de déficit estatural em crianças quilombolas $<5$ anos residentes em terras tituladas na Região Nordeste, Brasil, segundo características socioeconômicas e domiciliares (nível 2), 2011.

\begin{tabular}{|c|c|c|c|c|c|c|c|}
\hline \multirow[t]{2}{*}{ Variáveis } & \multicolumn{2}{|c|}{ E/I adequada } & \multicolumn{2}{|c|}{ Déficit estatural } & \multirow[t]{2}{*}{ Valor de $p$ * } & \multirow[t]{2}{*}{ RP (IC95\%) } & \multirow[t]{2}{*}{ Valor de $p * *$} \\
\hline & $\mathbf{n}$ & $\%$ & $\mathrm{n}$ & $\%$ & & & \\
\hline \multicolumn{8}{|c|}{ Renda total do domicílio } \\
\hline Quartil 4 & 142 & 91,0 & 14 & 9,0 & 0,123 & Referência (1) & \\
\hline Quartis 1 a 3 & 416 & 84,9 & 74 & 15,1 & & $0,72(0,48 ; 1,10)$ & 0,129 \\
\hline \multicolumn{8}{|c|}{$\begin{array}{l}\text { Condição ocupacional do chefe do } \\
\text { domicílio }\end{array}$} \\
\hline Desempregado & 480 & 84,1 & 91 & 15,9 & 0,081 & Referência (1) & \\
\hline Empregado & 315 & 89,2 & 38 & 10,8 & & $0,68(0,46 ; 0,99)$ & 0,042 \\
\hline Aposentado & 37 & 88,1 & 5 & 11,9 & & $0,75(0,30 ; 1,84)$ & 0,525 \\
\hline \multicolumn{8}{|c|}{ Domicílio com banheiro } \\
\hline Sim & 339 & 91,0 & 34 & 9,0 & $<0,001$ & Referência (1) & \\
\hline Não & 486 & 82,5 & 103 & 17,5 & & $1,92(1,30 ; 2,83)$ & $<0,001$ \\
\hline \multicolumn{8}{|c|}{ Domicílio com água tratada } \\
\hline Sim & 521 & 90,1 & 57 & 9,9 & $<0,001$ & Referência (1) & \\
\hline Não & 312 & 80,4 & 76 & 19,6 & & $1,99(1,41 ; 2,80)$ & $<0,001$ \\
\hline \multicolumn{8}{|c|}{ Forma de descarte do lixo } \\
\hline Adequada & 149 & 90,3 & 16 & 9,7 & 0,097 & Referência (1) & \\
\hline Inadequada & 686 & 85,0 & 121 & 15,0 & & $1,55(0,92 ; 2,60)$ & 0,101 \\
\hline \multicolumn{8}{|c|}{ Escoamento sanitário do domicílio } \\
\hline Adequado & 170 & 91,4 & 16 & 8,6 & 0,024 & Referência (1) & \\
\hline Inadequado & 658 & 84,7 & 119 & 15,3 & & $1,78(1,06 ; 3,00)$ & 0,030 \\
\hline \multicolumn{8}{|c|}{ Forma de iluminação do domicílio } \\
\hline Elétrica & 789 & 86,3 & 125 & 13,7 & 0,195 & Referência (1) & \\
\hline Outras fontes & 46 & 79,3 & 12 & 20,7 & & $1,51(0,84 ; 2,74)$ & 0,171 \\
\hline \multicolumn{8}{|c|}{ Quantidade de moradores do domicílio } \\
\hline$<5$ & 337 & 87,5 & 48 & 12,5 & 0,215 & Referência (1) & \\
\hline$\geq 5$ e $<11$ & 466 & 85,3 & 80 & 14,7 & & $1,18(0,82 ; 1,68)$ & 0,377 \\
\hline$\geq 11$ & 32 & 78,0 & 9 & 22,0 & & $1,76(0,86 ; 3,59)$ & 0,119 \\
\hline \multicolumn{8}{|c|}{$\begin{array}{l}\text { Quantidade de crianças }<5 \text { anos } \\
\text { morando no domicílio }\end{array}$} \\
\hline$<3$ & 728 & 86,9 & 110 & 13,1 & 0,041 & Referência (1) & \\
\hline$\geq 3$ & 107 & 79,9 & 27 & 20,1 & & $1,54(1,01 ; 2,34)$ & 0,046 \\
\hline \multicolumn{8}{|c|}{ Número de cômodos do domicílio } \\
\hline$\geq 3$ & 704 & 86,9 & 106 & 13,1 & 0,083 & Referência (1) & \\
\hline$<3$ & 131 & 81,4 & 30 & 18,6 & & $1,42(0,95 ; 2.14)$ & 0.087 \\
\hline
\end{tabular}

E/I: estatura-para-idade; IC95\%: intervalo de 95\% de confiança;

* Teste $\chi^{2}$ de Pearson;

** Teste de Wald. 
Tabela 4

Prevalência e razões de prevalência (RP) brutas de déficit estatural em crianças quilombolas $<5$ anos residentes em terras tituladas na Região Nordeste, Brasil, segundo características demográficas e de saúde da criança (nível 4), escolaridade dos pais e estado nutricional da mãe (nível 3), 2011.

\begin{tabular}{|c|c|c|c|c|c|c|c|}
\hline \multirow[t]{2}{*}{ Variáveis } & \multicolumn{2}{|c|}{ E/I adequada } & \multicolumn{2}{|c|}{ Déficit estatural } & \multirow[t]{2}{*}{ Valor de $p$ * } & \multirow[t]{2}{*}{ RP (IC95\%) } & \multirow[t]{2}{*}{ Valor de $p$ ** } \\
\hline & $\mathbf{n}$ & $\%$ & $\mathbf{n}$ & $\%$ & & & \\
\hline \multicolumn{8}{|l|}{ Estado } \\
\hline Bahia & 269 & 89,4 & 32 & 10,6 & $<0,001$ & Referência (1) & \\
\hline Maranhão & 293 & 79,6 & 75 & 20,4 & & $1,92(1,30 ; 2,90)$ & 0,002 \\
\hline Pernambuco & 139 & 89,7 & 16 & 10,3 & & $0,97(0,53 ; 1,77)$ & 0,923 \\
\hline Piauí & 104 & 89,7 & 12 & 10,3 & & $0,97(0,50 ; 1,89)$ & 0,936 \\
\hline Sergipe & 30 & 93,7 & 2 & 6,3 & & $0,59(0,14 ; 2,45)$ & 0,466 \\
\hline \multicolumn{8}{|l|}{ Sexo } \\
\hline Feminino & 439 & 86,4 & 69 & 13,6 & 0,698 & Referência (1) & \\
\hline Masculino & 396 & 85,3 & 68 & 14,7 & & $1,08(0,77 ; 1,51)$ & 0,636 \\
\hline \multicolumn{8}{|l|}{ Idade (anos) } \\
\hline$<1$ & 172 & 91,0 & 8 & 9,0 & 0,124 & Referência (1) & \\
\hline $1 \mathrm{e}<3$ & 306 & 84,8 & 55 & 15,2 & & $1,61(0,94 ; 2,74)$ & 0,080 \\
\hline $3 e<5$ & 357 & 84,8 & 64 & 15,2 & & $1,60(0,95 ; 2,71)$ & 0,076 \\
\hline \multicolumn{8}{|l|}{ Raça } \\
\hline Não negra & 47 & 82,5 & 10 & 17,5 & 0,531 & Referência (1) & \\
\hline Negra & 759 & 86,3 & 120 & 13,7 & & $0,78(0,41 ; 1,48)$ & 0,446 \\
\hline \multicolumn{8}{|c|}{ Diarreia nos últimos 7 dias } \\
\hline Não & 555 & 86,3 & 88 & 13,7 & 0,117 & Referência (1) & \\
\hline Sim & 74 & 79,6 & 19 & 20,4 & & $1,49(0,91 ; 2,45)$ & 0,113 \\
\hline \multicolumn{8}{|c|}{ Baixo peso ao nascer } \\
\hline Não & 666 & 88,5 & 87 & 11,5 & $<0,001$ & Referência (1) & \\
\hline $\operatorname{Sim}$ & 58 & 72,5 & 22 & 27,5 & & $2,38(1,49 ; 3,80)$ & $<0,001$ \\
\hline \multicolumn{8}{|c|}{$\begin{array}{l}\text { Escolaridade do chefe do } \\
\text { domicilio }\end{array}$} \\
\hline Quartil 4 & 154 & 90,1 & 17 & 9,9 & 0,107 & Referência (1) & \\
\hline Quartis 1 a 3 & 667 & 85,0 & 118 & 15,0 & & $1,51(0,91 ; 2,51)$ & 0,111 \\
\hline \multicolumn{8}{|c|}{ Escolaridade da mãe } \\
\hline Quartil 4 & 176 & 90,3 & 19 & 9,7 & 0,074 & Referência (1) & \\
\hline Quartis 1 a 3 & 509 & 84,8 & 91 & 15,2 & & $1,56(0,95 ; 2,55)$ & 0,079 \\
\hline \multicolumn{8}{|c|}{ Mãe com excesso de peso } \\
\hline Não & 361 & 83,6 & 71 & 16,4 & 0,123 & Referência (1) & \\
\hline Sim & 237 & 88,1 & 32 & 11,9 & & $0,72(0,48 ; 1,10)$ & 0,129 \\
\hline
\end{tabular}

E/I: estatura-para-idade; IC95\%: intervalo de 95\% de confiança;

* Teste $\chi^{2}$ de Pearson;

** Teste de Wald.

Quanto às variáveis demográficas, de saúde da criança, escolaridade dos pais e estado nutricional da mãe, a prevalência de déficit estatural foi 92\% maior no Maranhão, Brasil (IC95\%: 1,30; 2,90), quando comparado à Bahia (UF de referência). Em crianças nascidas com baixo peso, a prevalência foi 2,38 (IC95\%: 1,49; 3,80) vezes a de crianças que nasceram com peso adequado (Tabela 4).

No nível mais distal do modelo hierárquico final, crianças que residiam em domicílios quilombolas sem acesso à atenção básica apresentaram prevalência 63\% maior de déficit estatural (IC95\%: $1,11 ; 2,41)$ em comparação àquelas residentes em domicílios com acesso à atenção básica. No mesmo sentido, a prevalência de déficit estatural em crianças residentes em domicílio sem água tratada foi 2,09 (IC95\%: 1,42; 3,08) vezes a detectada em domicílios com acesso à água tratada. No nível mais 
proximal, após controlar por UF de residência, idade, ocorrência de diarreia e pelas variáveis que apresentaram significância estatística nos níveis anteriores, a prevalência de déficit estatural em crianças que nasceram com baixo peso foi 2,19 (IC95\%: 1,33; 3,61) vezes a prevalência daquelas que não apresentaram baixo peso no nascimento (Figura 1).

\section{Discussão}

A prevalência de déficit ponderal foi de 6,1\%, enquanto o excesso de peso atingiu 2,8\% da população estudada, valor inferior ao observado em crianças nordestinas na Pesquisa Nacional de Demografia e Saúde (PNDS), realizada em 2006 (7\%) 31.

Assim como a população quilombola, cujas estratégias de reconhecimento de direitos estão intimamente voltadas à garantia da posse de seus territórios, a população indígena é outro segmento populacional do país que possui histórico de iniquidades em saúde 32,33. Dados do I Inquérito Nacional de Saúde e Nutrição dos Povos Indígenas, realizado em 2008-2009, revelam semelhanças nas prevalências de déficit ponderal em relação ao presente estudo, tendo sido reportado que o agravo atingiu 4,1\% das crianças < 5 anos na Região Nordeste 21. Na população não quilombola da mesma região, o agravo acometeu 2,2\% das crianças, de acordo com a PNDS 31.

Por outro lado, os resultados do presente estudo evidenciaram elevada prevalência de déficit estatural (14,1\%), além de associações com variáveis de acesso à saúde, saneamento e estado de saúde da criança, assim como observado entre crianças indígenas da Região Nordeste em 2008-2009 (13,9\%) 21. A prevalência de déficit estatural na população não quilombola era de 5,9\% para as crianças nordestinas e de 6\% para a população brasileira em geral na mesma faixa etária em 2009 (POF 2009) 22. Na região do semiárido nordestino, área de reconhecida vulnerabilidade, 6,6\% dos pré-escolares apresentavam baixa E/I em 200534.

A investigação Chamada Nutricional Quilombola, realizada em 2006, abordou questões ligadas à condição social, política, cultural, econômica e de saúde da população quilombola no Brasil 35. Além de também ter sido observada prevalência elevada de déficit estatural (15\%) 20 , foi evidenciado que a maioria das famílias tinha baixa renda e reduzido acesso a serviços de saúde. Os dados desse estudo

\section{Figura 1}

Modelo hierárquico final para déficit estatural para idade entre crianças quilombolas $<5$ anos residentes em terras tituladas na Região Nordeste, Brasil, Pesquisa de Avaliação da Situação de Segurança Alimentar e Nutricional em Comunidades Quilombolas Tituladas, 2011.

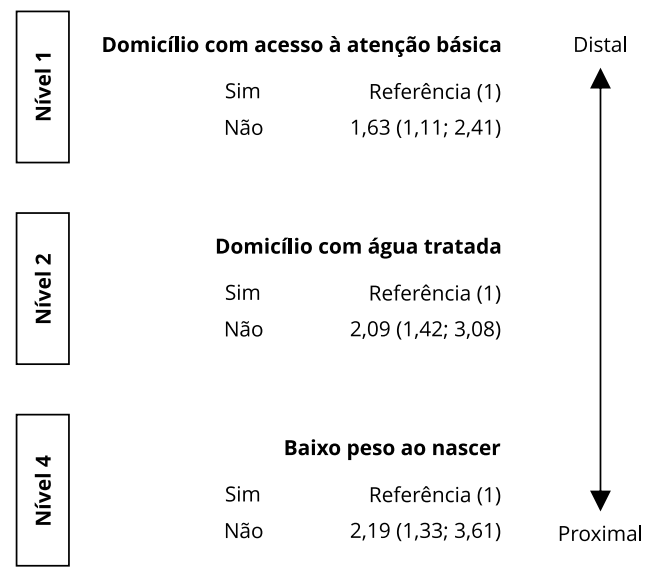


indicaram que mais de $80 \%$ dos chefes de família, além de viverem predominantemente em domicílios com esgoto a céu aberto, não haviam concluído o ensino fundamental 36 .

Apesar de não haver, na literatura, outros estudos sobre a saúde da população quilombola por região geográfica, é possível comparar os resultados do presente estudo com os de outros realizados em alguns estados do Nordeste. Ferreira et al. 11, em pesquisa realizada em Alagoas (UF não analisada no presente trabalho por não ter, à época, comunidades tituladas), observaram que $11,5 \%$ de quilombolas $<5$ anos apresentaram déficit estatural. Em adição, foi reportado que mais da metade da população investigada $(60,8 \%)$ pertencia à classe E, e $75,9 \%$ dos chefes do domicílio tinham baixa escolaridade ( $\leq 4$ anos de estudo). Cabe ressaltar que Alagoas apresenta o mais baixo Índice de Desenvolvimento Humano (IDH) $(0,631)$ da Região Nordeste 37 .

Na presente investigação, foi observada uma maior prevalência de déficit estatural no Maranhão quando este foi comparado com os demais estados avaliados. Trata-se do estado nordestino com maior número de comunidades quilombolas situadas em terras tituladas (22) 10. A elevada prevalência do agravo pode estar também relacionada ao IDH, pois, assim como Alagoas, o Maranhão detém um baixo valor desse índice $(0,639)$. Já Sergipe, que apresentou a menor prevalência $(6,2 \%)$, é o estado nordestino com o segundo maior IDH $(0,665) 37$.

Quilombolas e indígenas, segmentos da população brasileira historicamente marginalizados, têm demandado políticas públicas visando a reduzir as iniquidades sociais e o racismo estrutural 38. Dentre essas demandas, estão a necessidade de melhoria no acesso à saúde 39 , nas condições de habitação e saneamento 13 e a ampliação e qualificação da educação, valorizando as especificidades socioculturais 40,41. No entanto, apesar de algumas iniciativas, como a Política Nacional de Desenvolvimento Sustentável de Povos e Comunidades Tradicionais 42, fatores como o racismo estrutural e a exclusão social persistem como questões que dificultam a superação das iniquidades sociais por esse segmento populacional, com impacto negativo nos seus perfis de saúde, quando comparados à população geral do país.

Um exemplo pode ser encontrado no acesso à saúde por meio da rede de atenção básica. Por exemplo, a Política Nacional de Atenção Integral à Saúde da Criança (PNAISC) se baseia na promoção da saúde mediante estratégias como o incentivo ao aleitamento materno, promoção do crescimento e desenvolvimento integral e atenção a crianças com agravos prevalentes e em situação de vulnerabilidade. Nesse contexto, as EqSF têm importante papel, pois as ações de vigilância em saúde (visitas domiciliares, atuação do ACS) promovem a integralidade da assistência e a manutenção do cuidado à saúde da criança 43 . Ferreira \& Torres 2 demonstram a importância da promoção da saúde em comunidades quilombolas por meio da implementação de Estratégia Saúde da Família (ESF). Os autores investigaram diversos indicadores de saúde infantil antes e 12 meses após a implantação de uma unidade básica de saúde (UBS) em uma comunidade quilombola situada a $78 \mathrm{~km}$ da cidade de Maceió. Foram observados avanços expressivos nos indicadores investigados, o que foi atribuído a ações empreendidas pelos profissionais da equipe da ESF e ao maior acesso da população aos serviços disponibilizados na UBS.

É preocupante que mudanças na Política Nacional de Atenção Básica (PNAB), em vigência desde 2017, tenham o potencial de promover um desmonte das EqSF, o que pode impactar negativamente no acesso à saúde da população em geral, com consequências ainda mais negativas para grupos socialmente excluídos 44. Essa nova versão propõe a redução do número de ACS e de profissionais das EqSF, o que, junto a problemas como subfinanciamento, infraestrutura inadequada e precarização do trabalho, pode contribuir para aumentar as iniquidades em saúde de grupos socialmente vulneráveis, como quilombolas e indígenas. No presente estudo, quase metade das famílias não tinha acesso à atenção básica, sendo uma variável que permaneceu no modelo final como associada a um aumento na prevalência de déficit estatural.

A questão das iniquidades em saúde entre os quilombolas está intimamente associada a questões de saneamento básico. O Nordeste é a região com a segunda maior prevalência de domicílios não atendidos pela rede geral de abastecimento de água $(26,8 \%)$ e sem acesso à rede pública coletora de esgoto (72\%), ficando atrás apenas da Região Norte (42,9\% e 89,5\% respectivamente) 45 . De acordo com Andrade et al. 29 , a precariedade de saneamento básico e abastecimento de água atua como fator de risco para o desenvolvimento das enteroparasitoses que, por sua vez, são fatores de risco para diarreia e desnutrição. Entre 1997 e 2006, o estudo de Vasconcelos et al. 46 indicou que condições 
inadequadas de saneamento contribuíam para as prevalências elevadas de diarreia no Estado de Pernambuco. Na população indígena, $19,5 \%$ de crianças $<5$ anos apresentaram diarreia na semana anterior à entrevista, sendo este sintoma também associado com condições de saneamento 47 . Apesar de não ter apresentado associação com o déficit estatural, nossos resultados apontaram uma prevalência elevada do agravo (12,1\%).

Em comunidades quilombolas localizadas no sudoeste da Bahia, dois estudos reportaram elevadas frequências de domicílios que não tinham banheiro ou água canalizada, apresentavam esgoto a céu aberto e alta prevalência de indivíduos diagnosticados com parasitoses intestinais 13,14. A maioria dos domicílios das comunidades avaliadas no presente estudo não contava com banheiro, água tratada ou escoamento sanitário adequado. No modelo final, a falta de água tratada aumentou em duas vezes a prevalência de déficit estatural. É importante ressaltar que, em junho de 2020, o Senado Federal aprovou o novo marco legal do saneamento básico, o qual, entre outras diretrizes, prevê que a iniciativa privada realize serviços de saneamento em larga escala, o que pode aumentar o custo da água em áreas mais pobres e prejudicar as ações de saneamento em comunidades quilombolas.

O baixo peso ao nascer apresentou a maior associação com o déficit estatural, tanto na análise bruta quanto na ajustada. O baixo nível socioeconômico, que está refletido na baixa escolaridade e renda, tem sido reportado como fator de risco para a prematuridade e para o baixo peso ao nascer, que, por sua vez, impactam no crescimento e desenvolvimento da criança 48. Na amostra analisada, mais de $70 \%$ das mães não completaram o ensino fundamental, e a renda per capita era inferior a $\mathrm{R} \$$ 70,00 em aproximadamente $40 \%$ dos domicílios. A prevalência de baixo peso ao nascer observada nas crianças quilombolas $(11,2 \%)$ foi superior à encontrada na população nacional do estudo original $(9,8 \%)$ 49, evidenciando o ciclo de desigualdades ao qual a população quilombola está submetida na Região Nordeste.

O baixo peso ao nascer é um fator que aumenta a mortalidade neonatal, infantil e mesmo na fase adulta, afetando mais a população negra, quando comparada à branca 50,51. Em países pobres, como a Indonésia, o agravo aumenta substancialmente a prevalência de déficit estatural em crianças $<5$ anos 52 , o que também foi registrado para a população indígena no Brasil 21.

Ao mesmo tempo em que cabe reconhecer a relevância dos dados da Pesquisa de Avaliação para caracterização do perfil nutricional das crianças quilombolas, é preciso destacar que o estudo não envolveu o universo de coletividades quilombolas, haja vista que somente comunidades tituladas foram incluídas, deixando sem informação um enorme contingente formado pelas crianças residentes nas comunidades certificadas, mas não tituladas, existentes no país e, em especial, no Nordeste. Dessa forma, os resultados apresentados referem-se apenas às comunidades quilombolas tituladas, não podendo ser generalizados para o conjunto maior, que inclui não tituladas (certificadas). Tal fato se constitui numa limitação do presente estudo. É importante ressaltar que a certificação da comunidade já confere a implementação de medidas que visem à garantia de direitos a políticas públicas e programas sociais previstos em lei para a população quilombola 53. Existem, no Brasil, cerca de 3.400 comunidades quilombolas nessa situação, sendo a maioria (63\%) na Região Nordeste 54.

O estudo apresenta limitações adicionais que devem ser destacadas. Perdas relacionadas à impossibilidade de cálculo dos indicadores antropométricos para um conjunto de indivíduos, em face da ausência de dados de peso, estatura e idade, podem ter contribuído para a subestimação das prevalências de agravos nutricionais. Além disso, a disponibilização de dados pela Pesquisa de Avaliação não se deu juntamente com os respectivos totais de população das comunidades que participaram, o que compromete a caracterização dos perfis de ausência, recusa e/ou de pessoas não incluídas por dificuldades enfrentadas durante a pesquisa de campo. Trata-se de uma limitação que pode estar relacionada a viés de participação. Vale mencionar que a Pesquisa de Avaliação não coletou dados acerca de diagnóstico prévio de anemia ferropriva, variável que pode ser preditora de déficit estatural.

\section{Conclusões}

O presente estudo inova ao apresentar uma análise sobre o perfil nutricional de crianças quilombolas residentes na Região Nordeste com base na análise de um banco de dados de acesso público até o momento pouco explorado. 
O déficit estatural, indicativo de desnutrição crônica em estudos epidemiológicos, foi a condição mais prevalente entre as crianças investigadas, ao passo que as demais condições nutricionais foram consideradas de baixa magnitude. Os resultados do modelo final evidenciaram a centralidade do contexto social no qual a criança quilombola está inserida, o que afeta o seu crescimento linear. As análises conduzidas indicam, como amplamente sinalizado na literatura, que o crescimento infantil é fortemente influenciado pelas condições socioeconômicos às quais estão expostas as crianças, suas famílias e comunidades.

A análise da contribuição dos fatores socioeconômicos e demográficos à condição de saúde em comunidades quilombolas é uma importante estratégia para evidenciar como o processo saúde-doença se manifesta nesse segmento da população, o que inclui fatores históricos e socioeconômicos associados. As condições de vida da população quilombola são permeadas pelo racismo e pela exclusão, $o$ que se se manifesta em desigualdades nas mais variadas dimensões da vida social, incluindo educação, trabalho, habitação e saúde. Nesse cenário, a melhoria do estado nutricional das crianças quilombolas depende da implementação de ações de caráter intersetorial, fazendo-se necessárias amplas iniciativas de inclusão socioeconômica e política.

É importante que sejam realizadas mais investigações que analisem as condições de saúde da população quilombola, tanto em contextos regionais como em escala nacional. A literatura sobre o assunto é ainda escassa, o que limita as possibilidades de caracterização da situação geral de saúde, das demandas políticas e da produção dos elementos necessários para a implementação de estratégias de inclusão social que sejam econômica e culturalmente sensíveis aos contextos quilombolas. Inquestionavelmente, estratégias visando à ampliação da visibilidade social e política desse segmento populacional, como a evidenciação das iniquidades em saúde, são fundamentais para a concepção e implementação de políticas sociais que venham a reduzir as gritantes desigualdades étnico-raciais existentes no Brasil.

\section{Colaboradores}

F. J. Neves e A. A. Ferreira contribuíram na concepção do trabalho, análises, escrita do texto e aprovação da versão final. J. R. Welch contribuiu na concepção do trabalho, escrita do texto e aprovação da versão final.

\section{Informações adicionais}

ORCID: Félix de Jesus Neves (0000-0002-0700013X); Aline Alves Ferreira (0000-0001-50813462); James R. Welch (0000-0002-9094-5491).

\section{Agradecimentos}

Agradecemos os comentários de Ricardo Ventura Santos, da Escola Nacional de Saúde Pública Sergio Arouca, Fundação Oswaldo Cruz e do Museu Nacional, Universidade Federal do Rio de Janeiro, em uma versão preliminar deste texto. 


\section{Referências}

1. Popkin BM, Gordon-Larsen P. The nutrition transition: worldwide obesity dynamics and their determinants. Int J Obes 2004; 28:2-9.

2. Ferreira HS, Torres ZMC. Comunidade quilombola na Região Nordeste do Brasil: saúde de mulheres e crianças antes e após sua certificação. Rev Bras Saúde Mater Infant 2015; 15:219-29.

3. Pinheiro L, Fontoura NO, Querino AC, Bonneti A. Retrato das desigualdades de gênero e raça. 3a Ed. Brasília: Instituto de Pesquisa Econômica Aplicada; 2009.

4. Ministério do Desenvolvimento Social e Combate à Fome. Políticas sociais e Chamada Nutricional Quilombola: estudos sobre condições de vida nas comunidades e situação nutricional das crianças. Cadernos de Estudos: Desenvolvimento Social em Debate 2008; (9):1-142.

5. Florentino M, Amantino M. Uma morfologia dos quilombos nas Américas, séculos XVI -XIX. Hist Ciênc Saúde-Manguinhos 2012; 19:259-97.

6. Brasil. Decreto no 4.887 , de 20 de novembro de 2003. Regulamenta o procedimento para identificação, reconhecimento, delimitação, demarcação e titulação das terras ocupadas por remanescentes das comunidades dos quilombos de que trata o art. 68 do Ato das Disposições Constitucionais Transitórias. Diário Oficial da União 2003; 21 nov.

7. Marques CE, Gomes LA. Constituição de 1988 e a ressignificação dos quilombos contemporâneos. Limites e potencialidades. Rev Bras Ciênc Soc 2013; 28:137-255.

8. Williams DR. Race, socioeconomic status, and health the added effects of racism and discrimination. Ann N Y Acad Sci 1999; 896:173-88.

9. Freitas DA, Caballero AD, Marques AS, Hernández CIV, Antunes SLO. Saúde e comunidades quilombolas: uma revisão da literatura. Revista CEFAC 2011; 13:937-43.

10. Comissão Pró-Índio de São Paulo. Terras quilombolas - tituladas e em processo no INCRA. http://cpisp.org.br/publicacao/mapa-terrasquilombolas-tituladas-e-em-processo-no-in cra-2016/ (acessado em 16/Abr/2017).

11. Ferreira HS, Lamenha MLD, Xavier Junior AFS, Cavalcante JC, Santos AM. Nutrição e saúde das crianças das comunidades remanescentes dos quilombos no Estado de Alagoas. Rev Panam Salud Pública 2011; 30:51-8.

12. Bezerra VM, Medeiros DS, Gomes KO, Souza R, Giatti L, Steffens AP, Kochergin CN, Souza CL. Inquérito de saúde em comunidades quilombolas de Vitória da Conquista, Bahia, Brasil (Projeto COMQUISTA): aspectos metodológicos e análise descritiva. Ciênc Saúde Colet 2014; 191835-47.

13. Amorim MM, Tomazi L, Silva RAA, Gestinari RS, Figueiredo TB. Avaliação das condições habitacionais e de saúde da comunidade quilombola Boqueirão, Bahia, Brasil. Biosci J (Online) 2013; 29:1049-57.
14. Cabral-Miranda G, Dattoli VCC, Dias-Lima A. Enteroparasitos e condições socioeconômicas e sanitárias de uma comunidade quilombola do semiárido baiano. Rev Patol Trop 2010; 39:48-55.

15. Ferreira HS, Silva WO, Santos EA, Bezerra MKA, Silva BCV, Horta BL. Body composition and hypertension: a comparative study involving women from maroon communities and from the general population of Alagoas State, Brazil. Rev Nutr 2013; 26:539-49.

16. Gomes KO, Reis EA, Guimarães MDC, Cherchiglia ML. Utilização de serviços de saúde por população quilombola do Sudoeste da Bahia, Brasil. Cad Saúde Pública 2013; 29:1829-42.

17. Soares DA, Barreto SM. Sobrepeso e obesidade abdominal em adultos quilombolas, Bahia, Brasil. Cad Saúde Pública 2014; 30:341-54.

18. Guimarães RCR, Silva HP. Estado nutricional e crescimento de crianças quilombolas de diferentes comunidades do estado do Pará. Amazônica, Revista de Antropologia 2015; 7:186209.

19. Monego ET, Peixoto MRG, Cordeiro MM, Costa RM. (In)segurança alimentar de comunidades quilombolas do Tocantins. Segurança Alimentar e Nutricional 2010; 17:37-47.

20. Taddei JA, Colugnati F, Cobayashi F. Chamada nutricional: uma avaliação nutricional de crianças quilombolas de 0 a 5 anos. Cadernos de Estudos: Desenvolvimento Social em Debate 2008; (9):55-66.

21. Horta BL, Santos RV, Welch JR, Cardoso AM, Santos JV, Assis AMO, et al. Nutritional status of indigenous children: findings from the First National Survey of Indigenous People's Health and Nutrition in Brazil. Int J Equity Health 2013; 12:23.

22. Instituto Brasileiro de Geografia e Estatística. Pesquisa de Orçamentos Familiares 20082009: antropometria e estado nutricional de crianças, adolescentes e adultos no Brasil. Rio de Janeiro: Instituto Brasileiro de Geografia e Estatística; 2010.

23. Pinto AR, Borges JC, Novo MP, Pires PS. Quilombos do Brasil: segurança alimentar e nutricional em territórios titulados. Cadernos de Estudos: Desenvolvimento Social em Debate 2014; (20):1-212.

24. Pinto AR, Borges JC, Novo MP, Pires PS. Metodologia da pesquisa e avaliação da situação de segurança alimentar em comunidades quilombolas tituladas: lançando um novo olhar sobre os quilombos no Brasil. Cadernos de Estudo: Desenvolvimento Social em Debate 2014; (20):17-30.

25. Blössner M, Siyam A, Borghi E, Onyango A, Onis M. WHO Anthro Plus for personal computers manual: software for assessing growth of the world's children and adolescents. Geneva: World Health Organization; 2009. 
26. World Health Organization. Physical status: the use of and interpretation of anthropometry. Geneva: World Health Organization; 1995. (Report of a WHO Expert Committee).

27. Olinto MTA, Victora CG, Barros FC, Tomasi E. Determinantes da desnutrição Infantil em uma população de baixa renda: um modelo de análise hierarquizado. Cad Saúde Pública 1993; 9 Suppl 1:14-27.

28. Martins IS, Marinho SP, Oliveira DC, Araújo EAC. Pobreza, desnutrição e obesidade: inter-relação de estados nutricionais de indivíduos de uma mesma família. Ciênc Saúde Colet 2007; 12:1553-65.

29. Andrade EC, Leite ICG, Rodrigues VO, Cesca MG. Parasitoses intestinais: uma revisão sobre seus aspectos sociais, epidemiológicos, clínicos e terapêuticos. Revista de APS 2010; 13:23140.

30. Lestari ED, Hasanah F, Nugroho NA. Correlation between non-exclusive breastfeeding and low birth weight to stunting in children. Paediatrica Indonesiana 2018; 58:123-7.

31. Monteiro CA, Conde WL, Konno SC, Lima ALL, Silva ACF, Benício MHD. Avaliação antropométrica do estado nutricional de mulheres em idade fértil e crianças menores de cinco anos. In: Centro Brasileiro de Análise e Planejamento, Ministério da Saúde, organizador. Pesquisa Nacional de Demografia e Saúde da Criança e da Mulher - PNDS 2006: dimensões do processo reprodutivo e da saúde da criança. Brasília: Ministério da Saúde; 2009. p. 213-30.

32. Coimbra Jr. CEA, Santos RV. Saúde, minorias e desigualdade: algumas teias de inter-relações, com ênfase nos povos indígenas no Brasil. Ciênc Saúde Colet 2000; 5:125-32.

33. Ricardo B, Ricardo F. Povos indígenas no Brasil: 2006/2010. São Paulo: Instituto Socioambiental; 2011.

34. Monteiro CA, Conde WL, Konno SC. Análise do inquérito "Chamada Nutricional 2005" realizado pelo Ministério do Desenvolvimento Social e Combate à Fome e Ministério da Saúde. Cadernos de Estudos: Desenvolvimento em Debate 2006; (4):29-36.

35. Pereira LL, Bastos BM, Silva HO, Souza BO, Ayres G, Monteiro E, et al. Caracterização sociocultural das comunidades incluídas na Chamada Nutricional Quilombola. Cadernos de Estudos: Desenvolvimento em Debate 2008; (9):27-36.

36. Silva HO, Souza BO, Santos LMP. Diagnóstico das condições de vida nas comunidades incluídas na chamada nutricional quilombola. Cadernos de Estudos: Desenvolvimento em Debate 2008; (9):37-54.

37. Programa das Nações Unidas para o Desenvolvimento; Instituto de Pesquisa Econômica Aplicada. Desenvolvimento humano nas macrorregiões brasileiras. Brasília: Programa das Nações Unidas para o Desenvolvimento; Instituto de Pesquisa Econômica Aplicada; 2016.
38. Mattos H. "Remanescentes das comunidades dos quilombos": memória do cativeiro e políticas de reparação no Brasil. Revista USP 2006; 68:104-11.

39. Vieira ABD, Monteiro PS. Comunidade quilombola: análise do problema persistente do acesso à saúde, sob o enfoque da Bioética de Intervenção. Saúde Debate 2013; 37:610-8.

40. Arruti JMA. Políticas públicas para quilombos: terra, saúde e educação. In: Paula M, Heringer $\mathrm{R}$, organizadores. Caminhos convergentes: estado e sociedade na superação das desigualdades raciais no Brasil. Rio de Janeiro: Action Aid; 2009. p. 75-110.

41. Secretaria de Políticas de Promoção da Igualdade Racial. Programa Brasil Quilombola: diagnóstico de ações realizadas. Brasília: Secretaria de Políticas de Promoção da Igualdade Racial; 2012.

42. Shiraishi Neto J. Direitos dos povos e das comunidades tradicionais: declarações, convenções internacionais e dispositivos jurídicos definidores de uma política nacional. Manaus: Universidade do Estado do Amazonas; 2007.

43. Damasceno SS, Nóbrega VM, Coutinho SED, Reichert APS, Toso BRGO, Collet N. Saúde da criança no Brasil: orientação da rede básica à Atenção Primária à Saúde. Ciênc Saúde Colet 2016; 21:2961-73.

44. Melo EA, Mendonça MHM, Oliveira JR, Andrade GCL. Mudanças na Política Nacional de Atenção Básica: entre retrocessos e desafios. Saúde Debate 2018; 42:38-51.

45. Secretaria Nacional de Saneamento, Ministério do Desenvolvimento Regional. Sistema Nacional de Informações sobre Saneamento: $24^{\circ}$ diagnóstico dos serviços de água e esgotos - 2018. Brasília: Secretaria Nacional de Saneamento, Ministério do Desenvolvimento Regional; 2019.

46. Vasconcelos MJOB, Rissin A, Figueiroa JN, Lira PIC, Batista Filho, M. Fatores associados à diarreia em menores de cinco anos, no estado de Pernambuco, segundo inquéritos realizados em 1997 e 2006. Rev Saúde Pública 2018; 52:48.

47. Escobar AL, Coimbra Jr. CEA, Welch JR, Horta BL, Santos RV, Cardoso AM. Diarrhea and health inequity among Indigenous children in Brazil: results from the First National Survey of Indigenous People's Health and Nutrition. BMC Public Health 2015; 15:191.

48. Mahumud RA, Sultana M, Sarker AR. Distribution and determinants of low birth weight in developing countries. J Prev Med Public Health 2017; 50:18-28.

49. Sardinha LMV, Campos R, Pires OS, Jannuzzi P. Análise das condições de vida, segurança alimentar e nutricional e acesso a programas sociais em comunidades quilombolas tituladas. Cadernos de Estudos: Desenvolvimento Social em Debate 2014; (20):31-52. 
50. Silveira MF, Victora CG, Horta BL, Silva BGC, Matijasevich A, Barros FC. Low birthweight and preterm birth: trends and inequalities in four population-based birth cohorts in Pelotas, Brazil, 1982-2015. Int J Epidemiol 2019; 48 Suppl 1:i46-i53.

51. Coelho DM, Camelo LV, Giatti L, Chor D, Guimarães JMN, Mill JG, et al. Racial differences in the association between early socioeconomic position, birth weight, and arterial stiffness in adults from ELSA-Brasil. Ann Epidemiol 2019; 34:45-51.

52. Aryastami NK, Shankar A, Kusumawardani N, Besral B, Jahari AB, Achadi E. Low birth weight was the most dominant predictor associated with stunting among children aged 1223 months in Indonesia. BMC Nutrition 2017; $3: 16$.
53. Comissão Pró-Índio de São Paulo. O caminho da titulação das terras quilombolas. https:// cpisp.org.br/publicacao/o-caminho-da-titula cao-das-terras-quilombolas/ (acessado em 15/ $\mathrm{Abr} / 2017)$

54. Fundação Cultural Palmares. Quadro geral de Comunidades Remanescentes de Quilombos (CRQs). http://www.palmares.gov.br/wp-con tent/uploads/2015/07/TABELA-DE-CRQCOMPLETA-QUADRO-GERAL-3.pdf (acessado em 16/Abr/2017). 
Abstract

Quilombolas, or members of maroon communities in Brazil, are part of the country's ethnic/racial minorities exposed to health inequities, reflecting a historical process of harsh socioeconomic disadvantages. The study aimed to assess nutritional status and factors associated with stunting in quilombola children under five years of age living in land-deeded quilombola communities in Northeast Brazil. The study used secondary data from the Survey on Food and Nutritional Security in Land-Deeded Quilombola Communities (2011). The target outcomes were stunting (heightfor-age $<-2 z$ ), excess weight (weight-for-height $>$ $2 z$ ), and underweight (weight-for-age $<-2 z$ ). Chisquare test was used to assess the significance of differences between prevalence rates. Multivariate analysis used a hierarchical conceptual model on stunting. Prevalence rates for excess weight and underweight were $2.8 \%$ and $6,1 \%$, respectively. Stunting was diagnosed in $14.1 \%$ of the sample. The hierarchical model for stunting evidenced higher prevalence rates among children without access to primary healthcare $(P R=1.63 ; 95 \% C I$ : $1.11 ; 2.41)$ and safe water $(P R=2.09 ; 95 \% \mathrm{CI}$ : 1.42 ; 3.08$)$ and those with a history of low birthweight $(P R=2.19 ; 95 \% C I: 1.33 ; 3.61)$. The high prevalence of stunting showed that the quilombola' population in the Northeast experiences unfavorable health condition, reflecting lack of access to primary healthcare and precarious sanitation.

Ethnicity and Health; African Continental Ancestry Group; Nutrition Assessment; Malnutrition

\section{Resumen}

Los quilombolas constituyen parte de las minorías étnico-raciales del país que presentan inequidades en salud, reflejo de un proceso histórico que implicó grandes desigualdades socioeconómicas. El objetivo del trabajo fue evaluar el estado nutricional y los factores asociados con el déficit de estatura en niños quilombolas, menores de 5 años, residentes en comunidades quilombolas, ubicadas en la Región Nordeste del Brasil. Se utilizaron datos secundarios provenientes de la Pesquisa de Avaliação da Situação de Segurança Alimentar e Nutricional em Comunidades Quilombolas Tituladas (2011). Los resultados de interés fueron: déficit de estatura (estatura-para-edad $<-2 z$ ), exceso de peso (peso-para-estatura $>2 z$ ) y insuficiencia ponderal (peso-para-edad $<-2 z$ ). Se empleó la prueba chi-cuadrado para evaluar la significancia de las diferencias entre las prevalencias. El análisis multivariado consideró un modelo conceptual jerárquico sobre la insuficiencia de estatura. Las prevalencias de exceso de peso y déficit ponderal fueron $2,8 \%, 6,1 \%$, respectivamente. El déficit de estatura fue diagnosticado en un 14,1\% de la muestra. El modelo jerárquico de déficit de estatura evidenció mayores prevalencias de enfermedades entre niños que no tenían acceso a la atención básica $(R P=1,63$; IC95\%: 1,11; 2,41), al agua tratada $(R P=2,09$; IC95\%: 1,42; 3,08) y que nacieron con bajo peso ( $R P=2,1$; IC95\%: 1,33 ; 3,61). La elevada prevalencia de déficit de estatura muestra que la población quilombola en el Nordeste presenta condiciones de salud desfavorables, siendo reflejo de la falta de acceso a la atención básica en salud y de las precarias condiciones de saneamiento.

Origen Étnico y Salud; Grupo de Ascendencia Continental Africana; Evaluación Nutricional; Desnutrición
Recebido em 27/Mar/2020

Versão final reapresentada em 25/Nov/2020

Aprovado em 02/Dez/2020 